\title{
Các nhân tố ảnh hưởng đến chất lượng thông tin kế toán trên báo cáo tài chính của các doanh nghiệp niêm yết tại Thành phố Hồ Chí Minh
}

\section{Factors affect the quality of accounting information on financial statements of listed enterprises in Ho Chi Minh City}

\author{
Lê Hoàng Vân Trang ${ }^{*}$, Võ Văn Hiền ${ }^{2}$, Nguyễn Hoàng Thơ \\ 1,2,3 Trường Đại học Tiền Giang, Việt Nam \\ "Tác giả liên hệ, Email: lehoangvantrang @tgu.edu.vn
}

\begin{tabular}{|c|c|}
\hline THÔNG TIN & TÓM TÁ̀T \\
\hline $\begin{array}{l}\text { DOI: } 10.46223 / \mathrm{HCMCOUJS} \\
\text { econ.vi.15.2.246.2020 }\end{array}$ & $\begin{array}{l}\text { Mục đích của bài viết này là xác định mức đô ảnh hưởng } \\
\text { của các nhân tố bên trong đến chất lượng thông tin kế toán trên báo } \\
\text { cáo tài chính của doanh nghiệp niêm yết tại Thành phố Hồ Chí } \\
\text { Minh. Nghiên cứu hốn hợp được sử dụng trong bài báo, trong đó } \\
\text { nghiên cứu định tính được sự dụng trước với phương pháp tình }\end{array}$ \\
\hline Ngày nhận: 07/01/2020 & huống thông qua kỹ thuật thảo luận, phỏng vấn và sử dụng điểm \\
\hline Ngày nhận lại: 11/02/2020 & $\begin{array}{l}\text { bão hòa. Kêt quả cho thây có } 7 \text { nhân tô anh hưởng đê xây dựng mô } \\
\text { hình lý thuyêt, với thuế là nhân tố phát hiên mới - đăc thù khi thưc }\end{array}$ \\
\hline Duyệt đăng: 11/02/2020 & $\begin{array}{l}\text { hiện nghiên cứu tại Thành phố Hồ Chí Minh. Tiếp theo, nghiên cứu } \\
\text { định lượng được dùng đề kiểm định lại mô hình. Kết quả nghiên } \\
\text { cứu chỉ ra rằng có } 4 \text { nhân tố tác động đến chất lượng thông tin kế } \\
\text { toán trên báo cáo tài chính của doanh nghiệp niêm yết, được sắp } \\
\text { xếp theo mức độ ẩnh hưởng giảm dần: bộ mấy kế toán, nhà quản }\end{array}$ \\
\hline Tù khóa: & $\begin{array}{l}\text { lý, chứng từ kế toán và thuế. Trên cơ sở đó, nghiên cứu đưa ra một } \\
\text { số khuyến nghị nhăm nâng cao chất lượng thông tin kế toán tại các }\end{array}$ \\
\hline $\begin{array}{l}\text { áo cáo tài chính, chât lượng } \\
\text { lông tin, doanh nghiệp niêm } \\
\text { ôt, thông tin kế toân }\end{array}$ & $\begin{array}{l}\text { doanh nghiệp niêm yết trên địa bàn nghiên cứu, đồng thời đề xuất } \\
\text { hướng nghiên cứu cho tương lai. }\end{array}$ \\
\hline
\end{tabular}

\begin{abstract}
The objective of this paper is to identify the internal factors affecting the quality of accounting information on financial statements of Ho Chi Minh City listed enterprises. In this paper, a mixed study involved qualitative and quantitative researches. At first, qualitative research was used with case studies through discussion, interview and saturation point. The results showed that there were seven influential factors in building a theoretical model in which "tax" is a new specific factor to conduct research in Ho Chi Minh City. Then, quantitative research was used to retest the model. The research results indicated that there were four factors affecting the quality of accounting information on financial statements of listed enterprises

and being sorted by the level of diminishing influence: (1) accounting
\end{abstract}

financial statements, the quality of information, listed enterprises, accounting information 
networks, (2) managers, (3) accounting documents and (4) tax. Based on the study outcomes, we give some recommendations to the Ho Chi Minh City listed enterprises for improving the quality of accounting information and new directions in future research.

\section{1. Đặt vấn đề}

Thông tin kế toán (TTKT) được xem là một trong những công cụ hữu ích hỗ trợ cho các đối tượng sử dụng bên trong lẫn bên ngoài doanh nghiệp đưa ra các quyết định có liên quan. Do đó, chất lượng thông tin công bố trên báo cáo tài chính (BCTC) của các doanh nghiệp là vô cùng quan trọng, nó ảnh hưởng trực tiếp đến hiệu quả ra quyết định của người sử dụng thông tin.

Đối với các doanh nghiệp niêm yết (DNNY), TTKT trên BCTC đòi hỏi cao về chất lượng do: (1) người quan tâm đến TTKT trên $\mathrm{BCTC}$ rất đa dạng, phức tạp về trình độ và số lượng; (2) số lượng cổ đông lớn và biến động liên tục, việc theo dõi và phản ánh các nghiệp vụ vốn chủ sở hữu, phân phối lợi nhuận gặp nhiều khó khăn; (3) các TTKT đặc thù trên BCTC như thặng dư vốn cổ phần, cổ phiếu quỹ, lãi cơ bản trên cổ phiếu, việc tăng, giảm vốn chủ sở hữu trên thuyết minh BCTC và (4) việc công bố thông tin BCTC tuân thủ nghiêm ngặt hơn các doanh nghiệp thông thường.

Tuy nhiên, trong quá trình hoạt động, các DNNY thường công bố những BCTC với số liệu kế toán đã được "làm đẹp" thay vì số liệu thực của nó. Chính vì vậy, khi nền kinh tế có những biến động, không ít DNNY gặp khó khăn, thậm chí phá sản. Vẫn còn nguyên đó những bài học trên thế giới như Worldcom (1999), Enron (2001), Toshiba (năm 2015), ở Việt Nam có Viglacera Từ Sơn (2007). Đặc biệt, Thành phố Hồ Chí Minh (TP.HCM) thì có Bibica (2002), Bông Bạch Tuyết (2007), Viễn Đông (2011), gần đây nhất là Descon (2018). Điều này làm mất niềm tin công chúng vào thị trường chứng khoán cũng như gây khó khăn cho cơ quan quản lý Nhà nước.

Từ thực trạng trên, việc tìm hiểu và nghiên cứu các nhân tố ảnh hưởng chất lượng TTKT và đưa ra các khuyến nghị gia tăng chất lượng TTKT trên BCTC của các DNNY tại một trung tâm kinh tế lớn như TP.HCM là vấn đề quan trọng cần thực hiện.

\section{Cơ sở lý thuyết}

\subsection{Chất lự̛ng thông tin kế toán trên BCTC}

\section{Chất lượng thông tin (CLTT)}

Thông tin là sản phẩm của quá trình xử lý dữ liệu, làm cho dữ liệu có thêm ý nghĩa và giá trị trong những hoàn cảnh nhất định (Davenport \& Prusak, 1998). Thông tin cũng có lợi trong việc ra quyết định vì nó làm giảm sự không chắc chắn và tăng tri thức cho vấn đề được đề cập (Gelinas, Dull, \& Wheeler, 2011). Tương tự thông tin, chất lượng cũng có rất nhiều khái niệm khác nhau. Chất lượng là sự phù hợp nhu cầu (Juran, 1988). Theo Tổ chức tiêu chuẩn hóa quốc tế (ISO), ISO 9000:2005, chất lượng mang những đặc trưng cơ bản: thỏa mãn nhu cầu của người sử dụng, phù hợp với các nhu cầu khác nhau và được tiêu chuẩn hóa cụ thể. Từ hai khái niệm thông tin và chất lượng ở trên, có rất nhiều quan điểm khác nhau về CLTT. CLTT được định nghĩa như là thông tin đáp ứng những đặc điểm kỹ thuật hay đạt được các yêu cầu, sự 
mong đợi của người sử dụng thông tin (Khalil, Strong, Kahn, \& Pipino, 1999). Wang (1998) thì cho rằng CLTT là thông tin phù hợp cho việc sử dụng của người sử dụng thông tin. Theo đó, thông tin chất lượng là thông tin đảm bảo được bản chất, bối cảnh, biểu hiện và có thể truy cập được. Tóm lại, CLTT gồm các đặc tính cơ bản là chính xác, kịp thời, đầy đủ, dễ hiểu, so sánh được, có thể truy cập được và an toàn.

\section{Chất lượng TTKT trên BCTC}

TTKT trên BCTC về bản chất vẫn là thông tin, do đó chất lượng TTKT trên BCTC cũng cần được xây dựng dựa trên nền tảng CLTT. Theo Ủy ban chuẩn mực kế toán tài chính (FASB), chất lượng TTKT trên BCTC được chia làm 2 nhóm đặc điểm: (1) đặc điểm cơ bản gồm thích hợp và đáng tin cậy; và (2) đặc điểm thứ yếu gồm nhất quán và có thể so sánh được. Theo Hội đồng chuẩn mực kế toán quốc tế (IASB), các đặc điểm chất lượng TTKT trên BCTC gồm: có thể hiểu được, thích hợp, đáng tin cậy và có thể so sánh được. Dự án hội tụ IASB và FASB đã xác định chất lượng TTKT có các đặc điểm như thích hợp, trung thực, có thể so sánh, có thể kiểm chứng, kịp thời và có thể hiểu được. Theo Luật kế toán Việt Nam số 88/2015/QH13 và Chuẩn mực kế toán Việt Nam số 01, những thuộc tính của chất lượng TTKT trên BCTC là trung thực, khách quan, đầy đủ, kịp thời, có thể so sánh và kiểm chứng được. Riêng đối với DNNY, BCTC cần phải rõ ràng, đầy đủ các thông tin đặc thù như phân phối lợi nhuận, thặng dư vốn cổ phần, cổ phiếu quỹ và lãi cơ bản trên cổ phiếu.

\subsection{Các nghiên cứu liên quan chất lự̣ng TTKT trên BCTC}

Fathi (2013) nghiên cứu nhân tố quản trị công ty ảnh hưởng đến chất lượng TTKT trên BCTC của các DNNY tại Pháp, xác định 4 nhân tố ảnh hưởng gồm: (1) đặc điểm tài chính doanh nghiệp (đòn bẩy, lợi nhuận, tình trạng niêm yết); (2) đặc điểm của hội đồng quản trị; (3) chất lượng kiểm toán nội bộ và (4) cơ cấu sở hữu. Kết quả là các nhân tố trên đều có tác động tích cực đến chất lượng TTKT trên BCTC. Tuy nhiên, cơ cấu sở hữu có tác động ngược chiều. Abdullah, Almsafir, và Al-Smadi (2015) tìm ra mối quan hệ giữa chuẩn mực kế toán, quản trị doanh nghiệp, kiểm soát bên ngoài và tiêu chuẩn đạo đức với chất lượng TTKT trên BCTC dựa theo quan điểm của kiểm toán viên và quản lý ở Malaysia. Kết quả cho thấy các nhân tố đều ảnh hưởng đến chất lượng TTKT trên BCTC và chuẩn mực kế toán có mức ảnh hưởng cao nhất.

Tại Việt Nam, khi nghiên cứu về các doanh nghiệp tại Bình Định, T. A. Nguyen (2013) xác định các nhân tố bên trong như tổ chức công tác kế toán, tổ chức bộ máy kế toán,... có ảnh hưởng đến chất lượng TTKT. Vo và Tran (2013) qua phương pháp nghiên cứu tổng hợp lý thuyết và đúc kết từ thực tiễn, xác định các nhân tố có ảnh hưởng đến chất lượng TTKT trên BCTC như công tác tổ chức quản lý và người làm công tác kế toán. $\mathrm{N}$. T. H. Nguyen (2014) tiến hành chọn ngẫu nhiên 155 đối tượng trong $52 \mathrm{DNNY}$ để khảo sát thông qua hình thức phát phiếu khảo sát trực tiếp. Kết quả cho thấy nhân sự kế toán và nhà quản lý có ảnh hưởng đáng kể đến chất lượng TTKT. Tuy nhiên, nghiên cứu này chưa xây dựng các thang đo đo lường cụ thể cho các nhân tố tác động. Q. T. Pham (2016) khi đo lường mức độ tác động của các nhân tố đến chất lượng TTKT trình bày trên BCTC đã xác định 6 nhân tố bên trong doanh nghiệp như hệ thống chứng từ kế toán, tài khoản kế toán, sổ sách kế toán, nhà quản lý, bộ máy kế toán và mục tiêu lập $\mathrm{BCTC}$ có ảnh hưởng tích cực đến chất lượng TTKT. Khi nghiên cứu chất lượng TTKT với mẫu khảo sát 292 doanh nghiệp Việt Nam, T. T. Pham (2016) chỉ ra khá nhiều yếu 
tố bên trong lẫn bên ngoài có ảnh hưởng như tình trạng niêm yết, hiệu quả kiểm soát nội bộ, chất lượng phần mềm kế toán, đào tạo và bồi dưỡng, năng lực nhân viên kế toán, hỗ trợ từ phía nhà quản trị, kiểm toán độc lập, hành vi quản trị lợi nhuận và quy mô doanh nghiệp.

H. D. Nguyen (2010) cho rằng hệ thống kiểm soát chất lượng thông tin tài chính của DNNY gồm 6 thành phần có quan hệ tương hỗ nhau và cùng tác động đến chất lượng TTKT, đó là chuẩn mực kế toán, các quy định công bố BCTC, ban Giám đốc, ban kiểm soát, hệ thống kiểm soát nội bộ và kiểm toán độc lập. Le (2015) đề xuất mô hình nghiên cứu gồm hai nhóm nhân tố ảnh hưởng là nhóm đặc điểm tài chính và nhóm đặc điểm quản trị với 8 biến độc lập. Kết quả cho thấy, các nhân tố như đòn bẩy tài chính, lợi nhuận, công ty kiểm toán và hội đồng quản trị có ảnh hưởng đến chất lượng TTKT của các DNNY. Tuy nhiên, nghiên cứu chưa đề cập đến các nhân tố mang tính nguyên nhân bên trong chi phối việc lập BCTC của DNNY.

Tóm lại, theo các nghiên cứu trước, nhân tố ảnh hưởng đến chất lượng TTKT chủ yếu là nhóm nhân tố bên ngoài, nhóm đặc điểm tài chính, đặc điểm quản trị công ty mà chưa có nghiên cứu nào kiểm định sự ảnh hưởng của các nhân tố bên trong - mang tính nguyên nhân cho chất lượng TTKT trên BCTC một cách hệ thống. Nếu có, thì việc kiểm định các nhân tố ảnh hưởng này còn mang tính rời rạc như hệ thống kế toán (H. D. Nguyen, 2010), hội đồng quản trị hay nhà quản lý (Fathi, 2013; Le, 2015).

Để có thể đề xuất các giải pháp thiết thực và hợp lý nâng cao chất lượng TTKT trên BCTC, trước tiên cần phải xác định được các nhân tố ảnh hưởng bên trong thông qua nghiên cứu định tính để xây dựng mô hình lý thuyết và các thang đo phù hợp. Sau đó, sử dụng phương pháp định lượng để kiểm định lại mô hình lý thuyết và xác định được mức độ ảnh hưởng của các nhân tố đến chất lượng TTKT.

\section{Phương pháp nghiên cứu}

\subsection{Phưong pháp nghiên cứu định tính}

Được thực hiện bằng phương pháp nghiên cứu tình huống với kỹ thuật thảo luận và sử dụng điểm bão hòa (T. D. Nguyen, 2012).

Đối tuợng tham gia thảo luận: Các chuyên gia tài chính kế toán, giảng viên các trường đại học (thảo luận nháp), người làm kế toán và nhà quản lý tại DNNY (thảo luận chính thức).

Số lương mẫu: Từ 12 đến 15 tình huống, nếu lớn hơn thì số lượng thông tin thu thập sẽ khá nhiều, vượt quá khả năng theo dõi của nhà nghiên cứu (Xu, 2003). Bảng 1 thể hiện tóm tắt các tình huống trong nghiên cứu định tính:

\section{Bảng 1}

Mẫu cho nghiên cứu tình huống và kỹ thuật thu thập dữ liệu

\begin{tabular}{|c|l|l|}
\hline $\begin{array}{c}\text { Tình } \\
\text { huống }\end{array}$ & \multicolumn{1}{|c|}{ Đối tưọng phỏng vấn } & Kỹ thuật thu thập dữ liệu \\
\hline S1 & Kế toán CTY CP Gas Petrolimex (PGC) & Thảo luận nhóm (1) \\
\hline S2 & Kế toán trưởng CTY CP Gas Petrolimex (PGC) & Thảo luận nhóm (1) \\
\hline S3 & Kế toán trưởng CTY CP Viễn thông (VTC) & Thảo luận tay đôi \\
\hline S4 & Kế toán viên CTY CP đầu tư-thương mại (SMC) & Thảo luận nhóm (2) \\
\hline
\end{tabular}




\begin{tabular}{|c|l|l|}
\hline $\begin{array}{c}\text { Tình } \\
\text { huống }\end{array}$ & \multicolumn{1}{|c|}{ Đối tượng phỏng vấn } & Kỹ thuật thu thập dữ liệu \\
\hline S5 & Kế toán NHTMCP Sài Gòn Thương Tín (STB) & Thảo luận nhóm (2) \\
\hline S6 & Kế toán CTY giống cây trồng Miền Nam (SSC) & Thảo luận nhóm (2) \\
\hline S7 & Kế toán CTY CP cơ khí Xăng dầu (PMS) & Thảo luận tay đôi \\
\hline S8 & Trưởng phòng CTY CP Hàng Hải Sài Gòn (SHC) & Thảo luận tay đôi \\
\hline S9 & PGĐ CTY CP Nam Việt (NAV) & Thảo luận tay đôi \\
\hline S10 & Kế toán trưởng CTY CP nhiên liệu SG (SFC) & Thảo luận tay đôi \\
\hline S11 & Kế toán trưởng CTY Bê tông Châu Thới (BT6) & Thảo luận nhóm (3) \\
\hline S12 & Kế toán viên CTY Bê tông Châu Thới (BT6) & Thảo luận nhóm (3) \\
\hline & Giảng viên kế toán Đại học Kinh tế TP. HCM & Thảo luận nháp \\
\hline
\end{tabular}

Nguồn: Tổng hợp từ nghiên cứu

Quy trình chon mẫu: số lượng mẫu được xác định ở mức thông tin thu thập được hầu như không có gì khác biệt so với các mẫu trước đó. Sau đó tiếp tục chọn thêm một mẫu nhằm khẳng định điểm bão hòa. Nếu không phát hiện thêm thông tin gì mới thì sẽ ngừng lại. Quy trình thực hiện theo Hình 1:

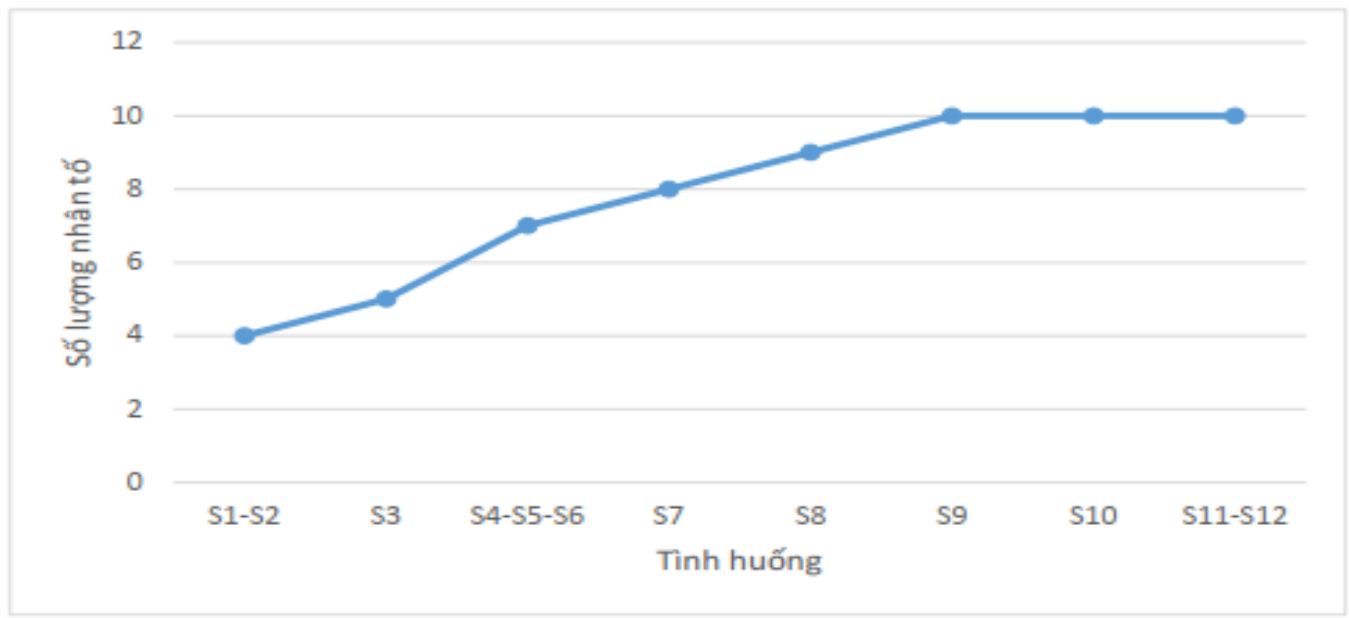

Hình 1. Quy trình chọn mẫu lý thuyết cho nghiên cứu tình huống

Thu thập và phân tích dũ liệu: Được thực hiện bằng dàn bài thảo luận, các câu hỏi trong dàn bài thảo luận được kế thừa từ các nghiên cứu trước và cơ sở lý thuyết.

Dựa vào mức độ tán thành của các tình huống, các nhân tố và thang đo chính thức được đưa vào mô hình nghiên cứu lý thuyết. Sau khi lấy ý kiến phản hồi, bảng câu hỏi khảo sát được hiệu chỉnh và sẵn sàng cho nghiên cứu định lượng.

\subsection{Phương pháp nghiên cúu định lự̣ng}

Đối tuợng thu thập dũ liệu: người phụ trách kế toán và nhà điều hành quản lý $(\mathrm{Xu}, 2003$, 2009; T. T. Pham, 2016).

Kich thước mẫu nghiên cứu: Theo T. D. Nguyen (2012), kích thước mẫu phụ thuộc vào nhiều yếu tố như phương pháp xử lý dữ liệu hay độ tin cậy cần thiết. 
Để thực hiện phân tích nhân tố khám phá EFA, kích thước mẫu sẽ được xác định bởi công thức: $\mathrm{n}=\mathrm{k}^{*}$ số biến quan sát (trong đó, $\mathrm{k}$ là tỷ lệ quan sát/biến quan sát, $\mathrm{k}$ thường là $5 / 1$ hoặc 10/1). Vậy, với số biến quan sát của mô hình nghiên cứu là 40 và hệ số $\mathrm{k}$ là $5 / 1$ thì kích thước mẫu tối thiểu là 200.

Để thực hiện phân tích hồi quy bội, kích thước mẫu thường được xác định theo công thức: $\mathrm{n} 50+8 \mathrm{k}$ (trong đó, $\mathrm{k}$ là số biến độc lập của mô hình). Trong nghiên cứu này, với số biến độc lập của mô hình nghiên cứu là 7 thì kích thước mẫu tối thiểu là 106 . Như vậy, xét các yêu cầu của phân tích EFA cũng như phân tích hồi quy bội, đồng thời xét giới hạn về mặt thời gian, số quan sát của nghiên cứu là 290 sẽ phù hợp.

Phương pháp chọn mẫu: Nghiên cứu sử dụng phương pháp chọn mẫu phi xác suất bởi sự thuận tiện về thời gian và chi phí. Bên cạnh đó, nếu chọn mẫu phi xác suất thì người nghiên cứu có thể thay thế phần tử tham gia vào mẫu, miễn sao phần tử đó thỏa mãn các tính chất cần có của phương pháp chọn mẫu phi xác suất. Hình thức chọn mẫu phi xác suất mà nghiên cứu thực hiện là chọn mẫu thuận tiện, nghĩa là nghiên cứu sẽ chọn những DNNY có thể tiếp cận được dễ் dàng.

Thu thập dũ liệu: Thực hiện thông qua bảng câu hỏi khảo sát. 290 phiếu khảo sát được phát đến các kế toán viên, kế toán trưởng và nhà quản lý DNNY. Bảng 2 thể hiện tóm tắt về DNNY được khảo sát:

\section{Bảng 2}

Bảng liệt kê DNNY khảo sát tại TP.HCM

\begin{tabular}{|c|l|c|c|c|}
\hline STT & Quận/Huyện & Số lưọng DNNY & Số phiếu khảo sát & $\begin{array}{c}\text { Số phiếu } \\
\text { trung bình/DNNY }\end{array}$ \\
\hline 1 & Bình Chánh & 9 & 35 & 3.9 \\
\hline 2 & Quận 3 & 7 & 28 & 4.0 \\
\hline 3 & Quận 1 & 8 & 33 & 4.1 \\
\hline 4 & Bình Tân & 15 & 54 & 3.6 \\
\hline 5 & Quận 5 & 12 & 46 & 3.8 \\
\hline 6 & Tân Phú & 6 & 25 & 4.2 \\
\hline 7 & Tân Bình & 7 & 23 & 3.3 \\
\hline 8 & Quận 11 & 8 & 29 & 4.3 \\
\hline 9 & Quận 10 & 4 & 17 & 3.8 \\
\hline & Tổng & $\mathbf{7 6}$ & $\mathbf{2 9 0}$ & \\
\hline
\end{tabular}

Nguồn: Tổng hợp từ nghiên cứu

Phân tích dũ liệu: Thực hiện thông qua thống kê mô tả, kiểm định độ tin cậy thang đo Cronbach's Alpha, phân tích nhân tố khám phá EFA và hồi quy tuyến tính đa biến với sự hỗ trợ của phần mềm SPSS 22.0.

Thang đo: Thang đo Likert 5 mức độ để đo lường giá trị các biến số. 


\section{Kết quả nghiên cứu và thảo luận}

\subsection{Kết quả nghiên cúu}

\subsubsection{Kết quả nghiên cứu định tính}

Thông qua thảo luận từ phần tử $\mathrm{S} 1$ đến $\mathrm{S} 12$, các dữ liệu thu thập được đủ để xác định các nhân tố có ảnh hưởng đến chất lượng TTKT của các DNNY. Kích thước mẫu cho nghiên cứu tình huống là $\mathrm{n}=12$, điểm bão hòa là $\mathrm{S} 10$, mẫu thu thập thỏa mãn điều kiện về số lượng đề nghị từ các nhà nghiên cứu trước đó.

Các nhân tố ảnh hưởng đến chất lượng TTKT thông qua thảo luận chính thức được trình bày theo Bảng 3 .

\section{Bảng 3}

Các tình huống đã khẳng định các nhân tố có ảnh hưởng đến chất lượng TTKT trên $\mathrm{BCTC}$ của DNNY

\begin{tabular}{|c|c|c|c|c|c|c|c|c|c|c|c|c|c|c|}
\hline STT & $\begin{array}{c}\text { Nhân tố } \\
\text { khám } \\
\text { phá }\end{array}$ & $\begin{array}{l}S \\
1\end{array}$ & $\begin{array}{l}S \\
2\end{array}$ & $\begin{array}{l}S \\
3\end{array}$ & $\begin{array}{l}S \\
4\end{array}$ & $\begin{array}{l}S \\
5\end{array}$ & $\begin{array}{l}S \\
6\end{array}$ & $\begin{array}{l}S \\
7\end{array}$ & $\begin{array}{l}\text { S } \\
8\end{array}$ & $\begin{array}{l}\text { S } \\
9\end{array}$ & $\begin{array}{c}S \\
10\end{array}$ & $\begin{array}{c}S \\
11\end{array}$ & $\begin{array}{c}S \\
12\end{array}$ & Tổng \\
\hline 1 & $\begin{array}{l}\text { Tài khoản } \\
\text { kế toán }\end{array}$ & 1 & 1 & 1 & 1 & 1 & 1 & 1 & 1 & 1 & 1 & 1 & 1 & 12 \\
\hline 2 & $\begin{array}{l}\text { Chứng từ } \\
\text { kế toán }\end{array}$ & 1 & 1 & 1 & 1 & 1 & 1 & 1 & 1 & 1 & 1 & 1 & 1 & 12 \\
\hline 3 & $\begin{array}{l}\text { Sổ sách } \\
\text { kế toán }\end{array}$ & 1 & 1 & 1 & 1 & 1 & 1 & 1 & 1 & 1 & 1 & 1 & 1 & 12 \\
\hline 4 & $\begin{array}{l}\text { Bộ máy } \\
\text { kế toán }\end{array}$ & 1 & 1 & 1 & 1 & 1 & 1 & 1 & 1 & 1 & 1 & 1 & 1 & 12 \\
\hline 5 & $\begin{array}{c}\text { Nhà quản } \\
\text { lý }\end{array}$ & & & & 1 & 1 & 1 & 1 & 1 & 1 & 1 & 1 & 1 & 9 \\
\hline 6 & $\begin{array}{c}\text { Mục tiêu } \\
\text { lập } \\
\text { BCTC }\end{array}$ & & & & 1 & 1 & 1 & 1 & 1 & 1 & 1 & 1 & 1 & 9 \\
\hline 7 & Thuế & & & & 1 & 1 & 1 & 1 & 1 & 1 & 1 & 1 & 1 & 9 \\
\hline 8 & $\begin{array}{c}\text { Hỗ trợ từ } \\
\text { nhà quản } \\
\text { trị }\end{array}$ & & & & & & & 1 & 1 & 1 & 1 & 1 & 1 & 6 \\
\hline 9 & $\begin{array}{l}\text { Hành vi } \\
\text { quản trị } \\
\text { lợi nhuận }\end{array}$ & & & 1 & & & & & & 1 & 1 & 1 & 1 & 5 \\
\hline 10 & $\begin{array}{c}\text { Năng lực } \\
\text { nhân viên } \\
\text { kế toán }\end{array}$ & & & & & & & & 1 & 1 & 1 & 1 & 1 & 5 \\
\hline \multicolumn{2}{|c|}{$\begin{array}{l}\text { Tổng cộng nhân } \\
\text { tố (từng phần tử) }\end{array}$} & 4 & 4 & 5 & 7 & 7 & 7 & 8 & 9 & 10 & 10 & 10 & 10 & \\
\hline
\end{tabular}

Nguồn: Tổng hợp từ dữ liệu nghiên cứu 
Để có cơ sở lựa chọn các nhân tố đưa vào mô hình nghiên cứu lý thuyết, nghiên cứu tiếp tục dựa vào dàn bài thảo luận từng đối tượng và tổng hợp, sắp xếp các nhân tố khám phá theo mức độ ảnh hưởng đến chất lượng TTKT:

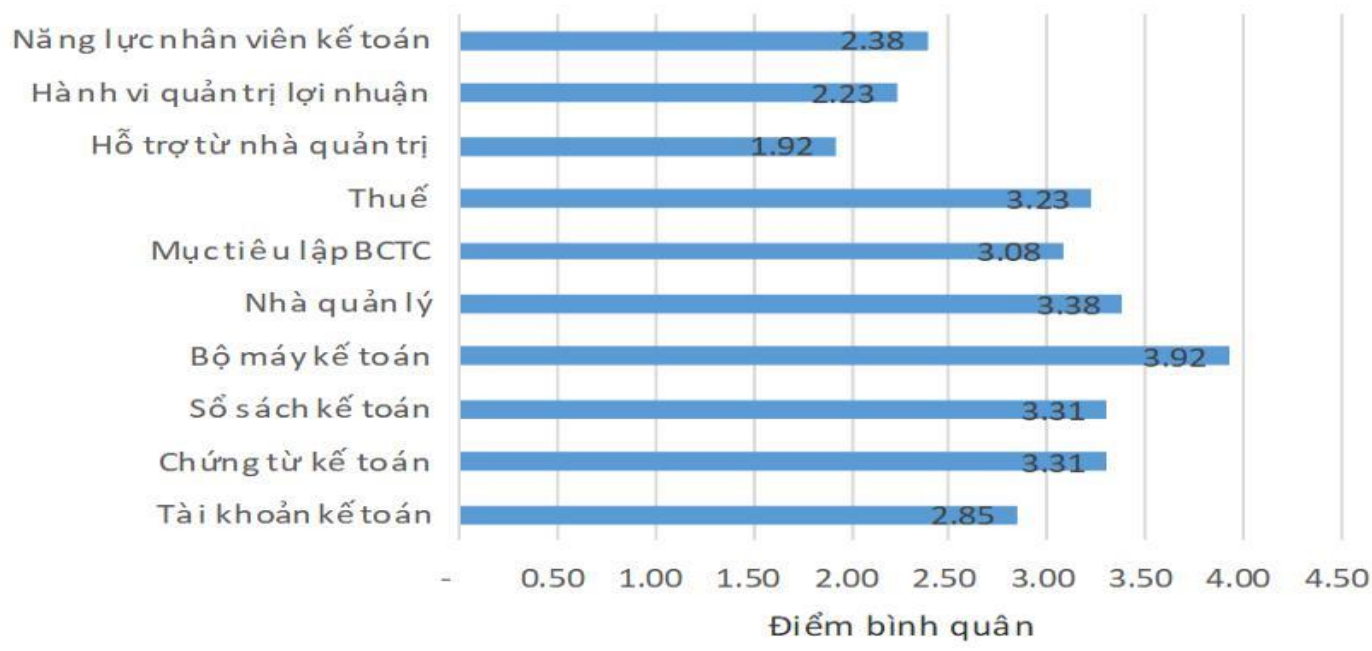

Hình 2. Các nhân tố ảnh hưởng chất lượng TTKT theo mức độ tác động Nguồn: Tổng hợp từ dữ liệu nghiên cứu

Xét theo mức độ tác động ở Hình 2, nhân tố tác động ở mức cao nhất là Bộ máy kế toán với 3.92 điểm. Tiếp theo là Nhà quản lý (3.38 điểm), hai nhân tố Chứng từ và Sổ sách kế toán cùng mức điểm là 3.31 . Thuế, Mục tiêu lập $\mathrm{BCTC}$ và Tài khoản kế toán có mức ảnh hưởng trung bình với số điểm lần lượt là $3.23 ; 3.08$ và 2.85 . Ba nhân tố còn lại có mức ảnh hưởng thấp là Năng lực nhân viên kế toán (2.38 điểm), Hành vi quản trị lợi nhuận (2.23 điểm) và Hỗ trợ từ nhà quản trị (1.92 điểm).

Để được lựa chọn vào mô hình nghiên cứu, các nhân tố khám phá phải được sự tán thành từ ý kiến của các chuyên gia với mức độ ảnh hưởng từ 2.5 điểm trở trên. Dựa vào tiêu chí đó, nghiên cứu loại bỏ 3 nhân tố là Năng lực nhân viên kế toán, Hành vi quản trị lợi nhuận và Hỗ trợ từ nhà quản trị ra khỏi mô hình nghiên cứu.

\section{Qua kết quả nghiên cứu định tính, nghiên cứu xác định:}

Nhân tố phát hiện mới: Thuế. Đây được xem là nhân tố đặc thù sau khi thực hiện nghiên cứu định tính tại TP.HCM, khác biệt so với các nghiên cứu trước. Thuế ở đây được xem là nhân tố bên trong ở khía cạnh là việc vận dụng chính sách thuế, các loại thuế và mức thuế suất đang hiện diện trên sổ sách kế toán của DNNY.

Các nhân tố còn lại của mô hình: Tài khoản kế toán, Chứng từ kế toán, Sổ sách kế toán, Bộ máy kế toán, Nhà quản lý và Mục tiêu lập BCTC tương đồng với các nghiên cứu trước ở phần cơ sở lý thuyết. Điều này phản ánh sự giao thoa, kế thừa của nghiên cứu này với các nghiên cứu trước trong cùng lĩnh vực.

Các nhân tố không được sư dụng trong mô hình: có 3 nhân tố là "Hỗ trợ từ nhà quản trị, Hành vi quản trị lợi nhuận và Năng lực nhân viên kế toán” không được tán thành bởi các chuyên gia trong phân tích tình huống mặc dù có trong các nghiên cứu trước cùng lĩnh vực. Lý do là tác động của các nhân tố này chưa rõ ràng hoặc rất khó có căn cứ để đo lường hoặc một số nhân tố đã được đề cập trong các nhân tố khám phá của nghiên cứu như: Hành vi quản trị 
lợi nhuận đã được đề cập trong nhân tố Mục tiêu lập BCTC; Hỗ trợ từ phía nhà quản trị đã được đề cập trong nhân tố Nhà quản lý và Năng lực nhân viên kế toán được đề cập trong Bộ máy kế toán.

Như vậy, mô hình nghiên cứu chính thức có 7 yếu tố (biến độc lập) như sau:

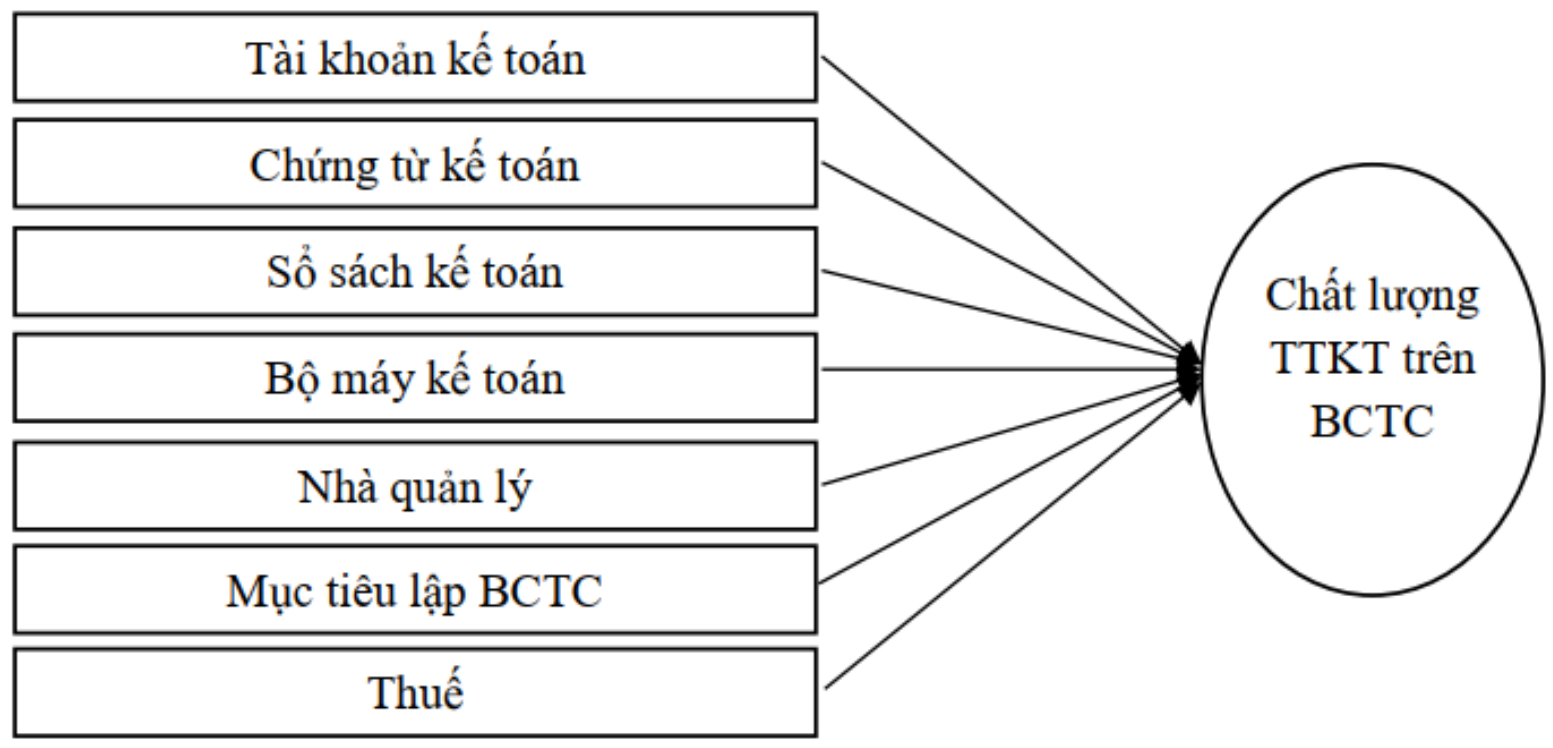

Hình 3. Mô hình nghiên cứu chính thức

Nguồn: Xây dựng từ kết quả nghiên cứu định tính

Sau khi xác định mô hình nghiên cứu, thông qua dàn bài thảo luận từng đối tượng, dữ liệu tiếp tục được phân tích, so sánh và kết nối để xây dựng giả thuyết, điều chỉnh thang đo đo lường các nhân tố cho phù hợp với các $\mathrm{DNNY}$ tại $\mathrm{TP}$. HCM, chuẩn bị cho phần nghiên cứu định lượng tiếp theo.

Tài khoản kế toán: Hệ thống tài khoản kế toán dựa vào chế độ kế toán, phù hợp với loại hình, lĩnh vực kinh doanh của doanh nghiệp, được mở đầy đủ và chi tiết. Thang đo Tài khoản kế toán là thang đo bậc 1 , được đo lường bởi 5 biến quan sát.

\section{H1: Tài khoản kế toán có ảnh hưởng tích cực đến chất lương TTKT trên BCTC}

Chứng tù kế toán: Chứng từ kế toán sử dụng tại doanh nghiệp theo đúng mẫu biểu quy định hiện hành, các nghiệp vụ kinh tế phát sinh phải có chứng từ được lập đầy đủ và kịp thời. Chứng từ được tổ chức luân chuyển một cách hợp lý và được sắp xếp lưu trữ thuận tiện khoa học. Thang đo Chứng từ kế toán là thang đo bậc 1 , được đo lường bởi 5 biến quan sát.

\section{H2: Chứng tù kế toán có ảnh hương tích cực đến chất lượng TTKT trên BCTC}

Sổ sách kế toán: Mẫu biểu sổ sách kế toán được áp dụng theo quy định kế toán, số liệu phải được ghi chép đúng và kịp thời. Việc áp dụng sổ sách phải phù hợp với loại hình kinh doanh của doanh nghiệp, sổ sách được mở chi tiết phục vụ cho việc ghi chép theo dõi và báo cáo. Các đối tượng giữ sổ và ghi sổ kế toán được phân quyền rõ ràng. Thang đo Sổ sách kế toán là thang đo bậc 1 , được đo lường bởi 5 biến quan sát.

\section{H3: Sổ sách kế toán có ảnh huởng tích cực đến chất lượng TTKT trên BCTC}


Bộ máy kế toán: Xây dựng bộ máy kế toán hoàn thiện, tổ chức bộ phận kế toán riêng biệt, đầu tư công nghệ thông tin cho bộ máy kế toán, nâng cao tinh thần trách nhiệm của từng cá nhân tham gia công tác kế toán. Nhân viên kế toán phải ký hợp đồng lao động với doanh nghiệp, nhằm tuân thủ và ràng buộc về trách nhiệm trong quá trình thu thập, xử lý và cung cấp thông tin. Thang đo Bộ máy kế toán là thang đo bậc 1 , được đo lường bởi 5 biến quan sát.

\section{H4: Bộ máy kế toán có ảnh hưởng tích cực đến chất lượng TTKT trên BCTC}

Nhà quản lý: Nhà quản lý phải hiểu biết nhất định về lĩnh vực kế toán tài chính, có khả năng đọc và hiểu thông tin $\mathrm{BCTC}$, quan tâm đến công tác kế toán của doanh nghiệp, dùng thông tin $\mathrm{BCTC}$ để ra các quyết định kinh tế. Thang đo Nhà quản lý là thang đo bậc 1 , được đo lường bởi 5 biến quan sát.

\section{H5: Nhà quản lý có ảnh hương tích cực đến chất lượng TTKT trên BCTC}

Muc tiêu lập BCTC: Xác định mục tiêu lập BCTC rõ ràng, đa dạng hóa đối tượng sử dụng thông tin. Với nhà quản lý doanh nghiệp, BCTC cung cấp thông tin để họ phân tích đánh giá và đưa ra các quyết định quản lý kịp thời, phù hợp cho sự phát triển của doanh nghiệp. Với các cơ quan Nhà nước như tài chính - ngân hàng, kiểm toán, thuế,... BCTC là tài liệu quan trọng trong việc kiểm tra giám sát, hướng dẫn, tư vấn cho doanh nghiệp thực hiện các chính sách, chế độ kinh tế tài chính. Thang đo Mục tiêu lập BCTC là thang đo bậc 1, được đo lường bởi 5 biến quan sát.

\section{H6: Mục tiêu lập BCTC có ảnh hương tích cực đến chất lượng TTKT trên BCTC}

Thuế: Tùy loại hình sản xuất kinh doanh mà mỗi doanh nghiệp vận dụng các chính sách thuế liên quan vào thực trạng của mình. Nhân viên kế toán cập nhật thường xuyên các chính sách thuế, ghi chép các nghiệp vụ liên quan thật rõ ràng trên sổ sách, tổ chức lưu trữ các hóa đơn, chứng từ phục vụ cho công tác kiểm tra, thanh tra. Việc nộp thuế vào ngân sách đầy đủ, đúng hạn là nghĩa vụ, thể hiện năng lực tài chính của doanh nghiệp. Thang đo Thuế là thang đo bậc 1 , được đo lường bởi 5 biến quan sát.

\section{H7: Thuế có ảnh hưởng tích cực đến chất lượng TTKT trên BCTC}

Chất luọng TTKT trên BCTC (nhân tố biến phụ thuộc): Chất lượng TTKT trình bày trên $\mathrm{BCTC}$ ảnh hưởng trực tiếp đến hiệu quả ra quyết định kỉnh tế của người sử dụng. Vì vậy thông tin kế toán phải được trình bày trung thực, khách quan, đầy đủ, kịp thời, có thể so sánh, kiểm chứng được, đặc biệt là các thông tin liên quan đến sự tăng, giảm vốn chủ sở hữu, phân phối lợi nhuận, lãi cơ bản trên cổ phiếu. Thang đo chất lượng TTKT là thang đo bậc 1, được đo lường bởi 5 biến quan sát.

\subsubsection{Kết quả nghiên cưu định lương}

Tổng số 290 phiếu khảo sát được phát ra, 261 phiếu khảo sát được thu về, trong đó có 5 phiếu không hợp lệ do trả lời cùng một mức độ cho tất cả các mục hỏi hoặc bị thiếu thông tin. Kết quả là có 256 phiếu khảo sát hợp lệ được sử dụng để làm dữ liệu cho nghiên cứu. Dữ liệu được nhập, mã hóa, làm sạch và phân tích thông qua phần mềm SPSS 22.0.

\section{Đánh giá độ tin cậy thang đo}

Hệ số tin cậy Cronbach's Alpha giúp loại đi những biến quan sát không đạt. Theo Hoang Trong và Chu (2008), khi Cronbach's Alpha từ 0.8 đến gần 1 thì thang đo lường là tốt, từ 0.7 đến gần 0.8 là sử dụng được. Dinh (2011) cho rằng, thang đo đạt chất lượng tốt khi hệ số Cronbach's Alpha > 0.6. 


\section{Bảng 4}

Hệ số Cronbach's Alpha

\begin{tabular}{|c|l|c|c|c|}
\hline STT & \multicolumn{1}{|c|}{ Tên biến } & Thang đo & $\begin{array}{c}\text { Cronbach's Alpha } \\
\text { của thang đo }\end{array}$ & Biến bị loại \\
\hline 1 & Tài khoản kế toán & TKKT & 0.321 & loại \\
\hline 2 & Chứng từ kế toán & CTKT & 0.887 & \\
\hline 3 & Số sách kế toán & SSKT & 0.865 & \\
\hline 4 & Bộ máy kế toán & BMKT & 0.860 & \\
\hline 5 & Nhà quản lý & NQL & 0.847 & \\
\hline 6 & Mục tiêu lập báo cáo tài chính & LBC & 0.486 & loại \\
\hline 7 & Thuế & THUE & 0.864 & \\
\hline 8 & Chất lượng thông tin kế toán & CLTT & 0.855 & \\
\hline
\end{tabular}

Nguồn: Kết quả phân tích SPSS (2019)

Kết quả đánh giá độ tin cậy của các biến quan sát thuộc thang đo CTKT, SSKT, BMKT, NQL, THUE, CLTT có Cronbach's Alpha > 0.8; hệ số tương quan biến tổng của các biến quan sát trong CTKT, SSKT, BMKT, NQL, THUE, CLTT $>0.3$. Như vậy, các thang đo này đều đạt yêu cầu để tiếp tục phân tích ở những bước sau. Riêng thang đo TKKT, LBC có Cronbach's Alpha $<0.6$ không đạt yêu cầu nên bị loại bỏ.

\section{Phân tích nhân tố khám phá EFA}

Kết quả thực hiện các kiểm định: $\mathrm{KMO}=0.941$; Bartlett's test có Sig. $=0.000<0.05$. Nghiên cứu cũng thể hiện $\%$ cumulative $=65.344 \%>50 \%$. Như vậy, kết quả các kiểm định trên đều đạt yêu cầu để phân tích nhân tố khám phá.

\section{Bảng 5}

KMO and Bartlett's Test

\begin{tabular}{|l|l|r|}
\hline \multicolumn{2}{|c|}{ Kaiser-Meyer-Olkin Measure of Sampling Adequacy. } & \multicolumn{1}{|c|}{.941} \\
\hline \multirow{2}{*}{$\begin{array}{l}\text { Bartlett's Test of } \\
\text { Sphericity }\end{array}$} & Approx. Chi-Square & 3460.891 \\
\cline { 2 - 3 } & Df & 231 \\
\cline { 2 - 3 } & Sig. & 0.000 \\
\hline
\end{tabular}

Nguồn: Kết quả phân tích từ SPSS, 2019

\section{Bảng 6}

Ma trận nhân tố đã xoay

\begin{tabular}{|l|c|c|c|c|}
\hline \multirow{2}{*}{} & \multicolumn{5}{|c|}{ Component } \\
\cline { 2 - 5 } & $\mathbf{1}$ & $\mathbf{2}$ & $\mathbf{3}$ & $\mathbf{4}$ \\
\hline CTKT1 & .666 & & & \\
\hline CTKT2 & .737 & & & \\
\hline CTKT3 & .693 & & & \\
\hline CTKT4 & .582 & & & \\
\hline CTKT5 & .763 & & & \\
\hline SSKT1 & .617 & & & \\
\hline SSKT4 & .638 & & & \\
\hline BMKT1 & & .642 & & \\
\hline BMKT2 & & .705 & & \\
\hline
\end{tabular}




\begin{tabular}{|c|c|c|c|c|}
\hline & \multicolumn{4}{|c|}{ Component } \\
\hline & 1 & 2 & 3 & 4 \\
\hline BMKT3 & & .734 & & \\
\hline BMKT4 & & .694 & & \\
\hline BMKT5 & & .671 & & \\
\hline NQL1 & & & & .637 \\
\hline NQL2 & & & & .702 \\
\hline NQL3 & & & & .671 \\
\hline NQL4 & & & & .693 \\
\hline NQL5 & & & & .635 \\
\hline THUE1 & & & .624 & \\
\hline THUE2 & & & .671 & \\
\hline THUE3 & & & .600 & \\
\hline THUE4 & & & .718 & \\
\hline THUE5 & & & .677 & \\
\hline
\end{tabular}

Extraction Method: Principal Component Analysis

Nguồn: Kết quả phân tích từ SPSS (2019)

Kết quả nhóm nhân tố dụa theo bảng ma trận xoay nhân tố như sau:

Nhân tố 1: gồm các biến CTKT1, CTKT2, CTKT3, CTKT4, CTKT5, SSKT1, SSKT4. Đặt tên cho nhân tố này là $C T K T$.

Nhân tố 2: gồm các biến BMKT1, BMKT2, BMKT3, BMKT4, BMKT5. Đặt tên cho nhân tố này là $B M K T$.

Nhân tố 3: gồm các biến THUE1, THUE2, THUE3, THUE4, THUE5. Đặt tên cho nhân tố này là $T H U E$.

Nhân tố 4: gồm các biến NQL1, NQL2, NQL3, NQL4, NQL5. Đặt tên cho nhân tố này là $N Q L$.

\section{Phân tích hồi quy tuyến tính đa biến}

\section{Bảng 7}

Các thông số thống kê trong mô hình hồi quy

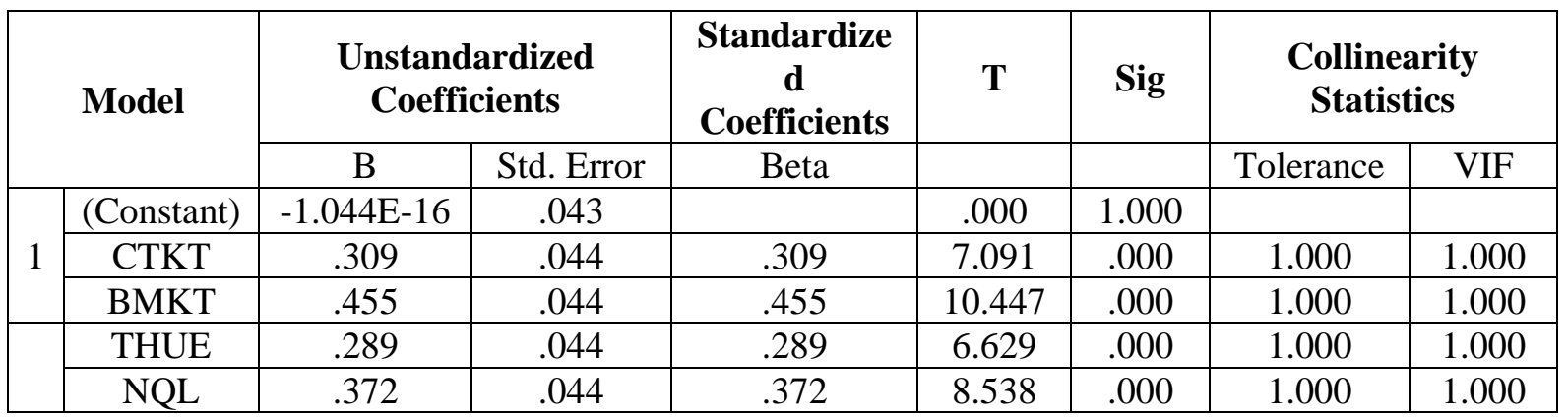

a. Dependent Variable: CLTT

Nguồn: Kết quả phân tích từ SPSS, 2019 
Phuong trình hồi quy tuyến tính đa biến được viết lại nhu sau:

$$
\mathrm{CLTT}=0.309 * \mathrm{CTKT}+0.455 * \mathrm{BMKT}+0.289 * \mathrm{THUE}+0.372 * \mathrm{NQL}
$$

Các biến CTKT, BMKT, THUE, NQL có Sig. $<0.01$ nên tương quan có ý nghĩa với CLTT, độ tin cậy là $99 \%$. Hệ số VIF < 5 nên không có hiện tượng đa cộng tuyến.

Bảng 8 thể hiện hệ số $\mathrm{R}^{2}$ hiệu chỉnh $=0.516$, có ý nghĩa là $51,6 \%$ sự thay đổi của biến phụ thuộc được giải thích bởi các biến độc lập. Còn lại 48,4\% là do các yếu tố tác động khác không được nghiên cứu trong mô hình này. Chỉ số Durbin Watson $=1.388<3$ nên không có hiện tượng tự tương quan.

\section{Bảng 8}

Hệ số tóm tắt mô hình

\begin{tabular}{|c|c|c|c|c|c|}
\hline Model & $\mathbf{R}$ & R Square & Adjusted R Square & Std. Error of the Estimate & Durbin-Watson \\
\hline 1 &, $724^{\mathrm{a}}$ & .524 & .516 & .69543691 & 1.388 \\
\hline
\end{tabular}

a. Predictors: (Constant), NQL, THUE, BMKT, CTKT

b. Dependent Variable: CLTT

Nguồn: Kết quả phân tích từ SPSS, 2019

Kết quả phân tích ANOVA cho thấy giá trị $\mathrm{F}=69.065$ với Sig. $<0.01$, có thể kết luận rằng mô hình đưa ra phù hợp với dữ liệu thực tế. Nói cách khác, các biến CTKT, $\mathrm{BMKT}$, THUE, NQL có tương quan tuyến tính với biến CLTT (độ tin cậy 99\%).

Phân tích tương quan thể hiện CTKT, BMKT, THUE và NQL đều có mức ý nghĩa Sig. $>0.05$. Như vậy, kiểm định này cho biết phương sai số dư không thay đổi.

Thông qua các hệ số như $\mathrm{R}^{2}$ hiệu chỉnh $=0.516$, Sig.F rất nhỏ, không có phương sai số dư thay đổi, không có hiện tượng đa cộng tuyến, nên có thể kết luận mô hình là phù hợp để giải thích các nhân tố ảnh hưởng đển chất lượng TTKT trên $\mathrm{BCTC}$ của các DNNY trên địa bàn TP.HCM.

\subsection{Thảo luận}

Mô hình hồi quy cho thấy có 4 nhân tố tác động đến chất lượng TTKT trên BCTC của DNNY tại TP. HCM. Cụ thể: CTKT với $\beta=0.309$; BMKT với $\beta=0.455$; THUE với $\beta=0.289$ và $\mathrm{NQL}$ với $\beta=0.372$. Tác động của các nhân tố trên đến CLTT trên $\mathrm{BCTC}$ được giải thích như sau:

CTKT tăng thêm 1 đơn vị thì chất lượng TTKT trên BCTC tăng 0.309 đơn vị.

BMKT tăng thêm 1 đơn vị thì chất lượng TTKT trên BCTC tăng 0.455 đơn vị.

THUE tăng thêm 1 đơn vị thì chất lượng TTKT trên BCTC tăng 0.289 đơn vị.

NQL tăng thêm 1 đơn vị thì chất lượng TTKT trên BCTC tăng 0.372 đơn vị.

Trong số các nhân tố ảnh hưởng, nhân tố BMKT có tác động mạnh nhất $(\beta=0.455)$, kế đến lần lượt là $\mathrm{NQL}(\beta=0.372), \mathrm{CTKT}(\beta=0.309)$ và THUE $(\beta=0.289)$.

Bộ máy kế toán có mức ảnh hưởng mạnh nhất đến chất lượng TTKT của DNNY với $\beta$ $=0.455$, vì đây là bộ phận trực tiếp ghi sổ và lập $\mathrm{BCTC}$. Năng lực của người làm kế toán là yếu 
tố quan trọng để các nghiệp vụ được hạch toán đúng và tạo ra các BCTC có chất lượng. Kết quả này phù hợp với nghiên cứu của $\mathrm{Q}$. T. Pham (2016) và Abdullah và cộng sự (2015). Một số nghiên cứu khác cũng chỉ ra bộ máy kế toán có ảnh hưởng mạnh đến chất lượng TTKT. Tuy nhiên chỉ dừng lại ở việc tổng hợp, phân tích công tác kế toán và đưa ra nhận định chứ chưa xác định mức độ ảnh hưởng (N. T. H. Nguyen, 2014; T. A. Nguyen, 2013; Vo \& Tran, 2013).

Tiếp đến, nhân tố có mức ảnh hưởng mạnh thứ hai đến chất lượng TTKT trên BCTC là nhà quản lý với $\beta=0.372$. Tuy họ không trực tiếp tham gia vào quá trình tạo ra thông tin kế toán, nhưng không thể phủ nhận sức ảnh hưởng của họ đến chất lượng TTKT. Nhà quản lý phải đánh giá, xem xét, kiểm tra được việc ghi chép và lập BCTC của bộ phận kế toán, góp phần hạn chế sai sót thông tin, số liệu kế toán, xác nhận và cho phép công bố các $\mathrm{BCTC}$ ra bên ngoài. Kết quả tương tự cũng được tìm thấy trong nghiên cứu của T. T. Pham (2016) và $\mathrm{Q}$. T. Pham (2016).

Nhân tố chứng từ kế toán với $\beta=0.309$ có mức ảnh hưởng mạnh thứ ba đến chất lượng TTKT. Chứng từ trong kế toán rất quan trọng, là yếu tố đầu vào cho quá trình xử lý thông tin kế toán. Do đó, chứng từ kế toán cần được lập, ghi chép, theo dõi và lưu trữ khoa học, đầy đủ. Sự ảnh hưởng của chứng từ kế toán đến chất lượng TTKT cũng được khẳng định trong nghiên cứu của $\mathrm{Q}$. T. Pham (2016) với $\beta=0.121$.

Nhân tố được xem là đặc thù, chưa được đề cập trong các nghiên cứu trước đó về lĩnh vực chất lượng TTKT trên BCTC là thuế (THUE) - xem xét ở khía cạnh nhân tố bên trong. Các nghiên cứu trước có đề cập đến thuế nhưng chỉ xem xét ở góc độ bên ngoài như áp lực từ cơ quan thuế (T. T. Pham, 2016).

Nhân tố thuế trong nghiên cứu của T. T. Pham (2016) được hiểu là áp lực từ cơ quan thuế với $\beta=-0.143$, mức độ ảnh hưởng mạnh thứ 3 , tác động nghịch chiều đến CLTT trên BCTC. Trong nghiên cứu này, thuế được hiểu là việc vận dụng chính sách thuế vào công tác kế toán tại doanh nghiệp. Khi ghi chép và lập $\mathrm{BCTC}$, việc tuân thủ đúng chính sách thuế sẽ phản ánh đúng bản chất thông tin kế toán. Điều này được ủng hộ bởi $\mathrm{Q}$. T. Pham (2016) với $\beta$ (thuế) $=0.167$.

Nghiên cứu đã xây dựng một mô hình lý thuyết về chất lượng TTKT tại các DNNY, cụ thể là mô hình các nhân tố bên trong mang tính nguyên nhân cho CLTT trên BCTC gồm bộ máy kế toán, nhà quản lý, chứng từ kế toán và thuế, trong đó, thuế được xem là nhân tố mới. Nghiên cứu định lượng kiểm định lại mô hình lý thuyết và xác định mức độ ảnh hưởng cụ thể của từng nhân tố. Ngoài ra, kết quả của nghiên cứu giúp khẳng định kết quả của các nghiên cứu trước thông qua 3 nhân tố còn lại. Từ đó, đề xuất các khuyến nghị nhằm nâng cao chất lượng TTKT tại các DNNY trên địa bàn.

Tuy nhiên, việc lượng hóa chất lượng TTKT trên BCTC trong nghiên cứu này chủ yếu với đối tượng khảo sát là người cung cấp thông tin như kế toán viên và quản lý bên trong DNNY. Nghiên cứu chưa khảo sát mức độ chấp nhận (hài lòng) về chất lượng TTKT của đối tượng bên ngoài hay gọi là người sử dụng thông tin để có cái nhìn toàn diện hơn về chất lượng thông tin. Mặc dù nghiên cứu phù hợp về lý thuyết và ý nghĩa nhưng mẫu nghiên cứu chưa bao quát hết các DNNY tại TP. HCM, chưa đi sâu phân tích kiểm định có hay không sự khác biệt về chất lượng TTKT giữa các DNNY có ngành nghề kinh doanh hay chế độ kế toán áp dụng khác nhau. Hệ số $\mathrm{R}^{2}=0.516$, tức là chỉ $51.6 \%$ sự thay đổi của chất lượng TTKT được giải thích bởi các biến độc lập, còn lại $48.4 \%$ là các nhân tố khác, có thể là chất lượng phần mềm kế toán, 
nhân viên kế toán, đào tạo và bồi dưỡng,... Những hạn chế trên đây cũng chính là cơ sở cho các nghiên cứu trong tương lai.

\section{Kết luận và khuyến nghị}

\subsection{Kết luận}

Nghiên cứu này kế thừa các kết quả nghiên cứu trước đó về chất lượng TTKT, kết hợp với dữ liệu thu thập từ nghiên cứu định tính để xác định các nhân tố ảnh hưởng và xây dựng mô hình lý thuyết phù hợp với các DNNY tại TP.HCM. Nghiên cứu định lượng kiểm định lại mô hình thông qua Cronbach's Alpha, EFA và phân tích hồi quy chỉ ra rằng có bốn nhân tố ảnh hưởng tích cực đến chất lượng TTKT trên BCTC là Bộ máy kế toán, Nhà quản lý, Chứng từ kế toán và Thuế. Các nhân tố này phát triển mạnh theo hướng tích cực thì chất lượng TTKT sẽ gia tăng. Thuế là nhân tố mới - đặc thù trong nghiên cứu này. Dựa vào đó nghiên cứu đề xuất các khuyến nghị nhằm nâng cao chất lượng TTKT trên BCTC của DNNY tại TP.HCM.

\subsection{Khuyến nghị}

Bộ máy kế toán: Cần hoàn thiện hơn bộ máy kế toán, chú trọng vào công tác đào tạo bồi dưỡng trình độ chuyên môn của bộ phận kế toán, nâng cao tinh thần trách nhiệm và tạo điều kiện cho kế toán viên tham gia tập huấn cập nhật kiến thức nghiệp vụ khi thay đổi chính sách. Doanh nghiệp cũng cần đầu tư ứng dụng công nghệ thông tin vào tổ chức công tác kế toán như phần mềm kế toán Misa, giúp cải thiện các khâu trong lập BCTC. Nhân viên kế toán còn phải nâng cao kỹ năng đọc hiểu các $\mathrm{BCTC}$ chứ không chỉ dừng lại ở việc lập $\mathrm{BCTC}$, việc lượng hóa chất lượng thông tin BCTC của DNNY đảm bảo các tiêu chí cơ bản của thông tin như thích hợp, trung thực, dễ hiểu, so sánh được và kịp thời, giúp cho các đối tượng sử dụng có thể đánh giá được chất lượng thông tin BCTC.

Nhà quản lý: Cần nâng cao mức độ hiểu biết nhất định của họ về lĩnh vực kế toán. Nhà quản lý phải biết đánh giá, xem xét, kiểm tra được việc ghi chép và lập BCTC của bộ phận kế toán, góp phần hạn chế sai sót thông tin, số liệu kế toán do bộ phận này cung cấp. Bên cạnh đó nhà quản lý cần quan tâm hơn đến nội dung thông tin trình bày trên $\mathrm{BCTC}$ để ra quyết định liên quan. Nhà quản lý biết sử dụng và hiểu được thông tin từ $\mathrm{BCTC}$ thì sẽ tạo ra độ tin cậy cao cho các đối tượng sử dụng BCTC.

Chứng tù kế toán: Tổ chức hệ thống chứng từ kế toán tốt có ảnh hưởng rất lớn và gián tiếp hỗ trợ cho việc ghi chép sổ sách, theo dõi và kiểm tra thông tin, số liệu trên $\mathrm{BCTC}$, việc này đòi hỏi phải được thực hiên một cách khoa học, bài bản. Tổ chức chứng từ kế toán phải căn cứ vào quy mô sản xuất, trình độ tổ chức quản lý của doanh nghiệp để xác định số lượng, loại chứng từ thích hợp. Các luật, quy định, thông tư liên quan cũng là cơ sở tăng cường tính pháp lý của chứng từ.

Thuế: Chính sách thuế thường xuyên thay đổi. Vì vậy, nhân viên kế toán phải được tập huấn và cập nhật kiến thức về thuế. Tuân thủ các chính sách thuế, hướng dẫn của cơ quan thuế sẽ đảm bảo việc hạch toán các nghiệp vụ liên quan và trình bày rõ ràng trên BCTC. Tùy loại hình kinh doanh, những loại hàng hóa dịch vụ được miễn, giảm thuế mà các DNNY phải quản lý, theo dõi, ghi chép riêng để thực hiện đúng nghĩa vụ đối với Nhà nước. Hạn chế thực trạng trốn thuế, bị truy thu thuế, bị phạt nộp chậm ở các DNNY. Cần lưu trữ có hệ thống, đầy đủ các hóa đơn, chứng từ liên quan để phục vụ cho công tác thanh tra của cơ quan thuế. 


\section{Tài liệu tham khảo}

Abdullah, Z. I. B., Almsafir, M. K., \& Al-Smadi, A. A. M. (2015). Transparency and reliability in financial statement: Do they exist? Evidence from Malaysia. Open Journal of Accounting, 4(4), 29-43.

Bộ Tài chính. (2002). Chuẩn mục số 01: Chuẩn mục chung [Standard No. 01: General standard]. Retrieved December 31, 2002, from https://atconline.vn/threads/chuan-mucke-toan-so-1-chuan muc-chung.5/

Davenport, T. H., \& Prusak, L. (1998). Working knowledge: How organizations manage what they know. Brighton, MA: Harvard Business Press.

Dinh, H. P. (2011). Phưong pháp nghiên cứu định lương \& nhũng nghiên cúu thục tiễn trong kinh tế phát triển - nông nghiệp [Quantitative research method \& practical research in development economics - agriculture]. Ho Chi Minh, Vietnam: NXB Phương Đông.

Fathi, J. (2013). The determinants of the quality of financial information disclosed by French listed companies. Mediterranean Journal of Social Sciences, 4(2), 319-336.

Financial Accounting Standards Board (FASB). (2010). Qualitative characteristics of useful financial information. In Conceptual framework for financial reporting. Norwalk, CO: Financial Accounting Foundation.

Gelinas, U. J., Dull, R. B., \& Wheeler, P. (2011). Accounting information systems. Boston, MA: Cengage learning.

Hoang Trong \& Chu, N. N. M. (2008). Phân tích dũ liệu nghiên cúu với SPSS [Analyzing research data with SPSS]. Ho Chi Minh, Vietnam: NXB Hồng Đức.

IASB. (2010). The conceptual framework for financial reporting. London, UK: International Accounting Standards Board.

Juran, J. M. (1988). Juran on planning for quality. New York, NY: Collier Macmillan.

Khalil, O. E., Strong, D. M., Kahn, B. K., \& Pipino, L. L. (1999). Teaching information quality in information systems undergraduate education. Informing Science: The International Journal of an Emerging Transdiscipline, 2, 53-60.

Le, H. T. M. (2015). Minh bạch thông tin tài chính của các công ty niêm yêt trên thị truờng chúng khoán Việt Nam [Transparency of financial information of companies listed on Vietnam's stock market] (Doctoral dissertation, University of Economics, Ho Chi Minh City, Vietnam). Retrieved October 20, 2018, from https://thegioiluat.vn/bai-viet-hocthuat/LUAN-VAN-TIEN-SI--MINH-BACH-THONG-TIN-TAI-CHINH-CUA-CACCONG-TY-NIEM-YET-TREN-THI-TRUONG-CHUNG-KHOAN-VIET-NAM-10488/

Nguyen, H. D. (2010). Hệ thống kiểm soát sụ minh bạch thông tin tài chính công bố của các công ty niêm yết tại Việt Nam [The system controls the transparency of financial information disclosed by listed companies in Vietnam] (Doctoral dissertation, University of Economics, Ho Chi Minh City, Vietnam). Retrieved October 26, 2018, from http://digital.lib.ueh.edu.vn/handle/UEH/42417 
Nguyen, N. T. H. (2014). Tiêu chuẩn đánh giá và các yếu tố ảnh hưởng đến chất lượng thông tin kế toán [Evaluation criteria and factors affecting the quality of accounting information]. Tạp chí Kế toán \& Kiểm toán, 131, 30-33.

Nguyen, T. A. (2013). Công tác kế toán trong các DNNVV trên địa bàn tỉnh Bình Định [Accounting work in SMEs in Binh Dinh province] (Master's thesis, University of Da Nang, Da Nang City, Vietnam). Retrieved September 25, 2018, from http://luanvan.net.vn/luan-van/cong-tac-ke-toan-trong-cac-doanh-nghiep-nho-va-vuatren-dia-ban-tinh-binh-dinh-51384/

Nguyen, T. D. (2012). Phưong pháp nghiên cứu khoa học trong kinh doanh [Scientific research method in business]. Hanoi, Vietnam: NXB Lao động - Xã hội.

Pham, Q. T. (2016). Các nhân tố tác động đến chất lượng thông tin báo cáo tài chính trong các doanh nghiệp tại Việt Nam [Factors affecting the quality of financial reporting information in enterprises in Vietnam] (Doctoral dissertation, University of Economics, Ho Chi Minh City, Vietnam). Retrieved September 20, 2018, from https://www.academia.edu/35437807/LA01_048_C\%C3\%A1c_nh\%C3\%A2n_t\%E1\% BB\%91_t\%C3\%A1c_\%C4\%91\%E1\%BB\%99ng_\%C4\%91\%E1\%BA\%BFn_ch\%E1\% BA\%A5t_1\%C6\%B0\%E1\%BB\%A3ng_th\%C3\%B4ng_tin_b\%C3\%A1o_c\%C3\%A1o_t \%C3\%A0i_ch\%C3\%ADnh_trong_c\%C3\%A1c_doanh_nghi\%E1\%BB\%87p_t\%E1\%B A\%A1i_Vi\%E1\%BB\%87t_Nam_pdf

Pham, T. T. (2016). Đo lường mức độ tác động của các nhân tố đến chất lượng thông tin trình bày trên báo cáo tài chính của các doanh nghiệp nhỏ và vìa ở Thành phố Hồ Chí Minh [Measuring the impact of factors on the quality of information presented on financial statements of SMEs in Ho Chi Minh City] (Master's thesis, University of Economics, Ho Chi Minh City, Vietnam). Retrieved September 25, 2018, from http://digital.lib.ueh.edu.vn/handle/UEH/44176

Quốc hội. (2015). Luật kế toán [Law on accounting]. Retrieved November 20, 2015, from https://thuvienphapluat.vn/van-ban/ke-toan-kiem-toan/Luat-ke-toan-2015-298369.aspx

Vo, N. V., \& Tran, H. T. T. (2013). Một số ý kiến về chất lượng báo cáo tài chính của DNNVV ở Việt Nam [Some comments on the quality of SMEs' financial statements in Vietnam]. Tạp chi Kinh tế - Kỹ thuật, 6(2), 3-12.

Wang, R. Y. (1998). A product perspective on total data quality management. Communications of the ACM, 41, 58-65.

Wang, R. Y., \& Strong, D. M. (1996). Beyond accuracy: What data quality means to data consumers. Journal of management information systems, 12(4), 5-33.

$\mathrm{Xu}, \mathrm{H}$. (2003). Critical success factors for accounting information systems data quality (Doctoral dissertation). University of Southern Queensland, Australia.

$\mathrm{Xu}, \mathrm{H}$. (2009). Data quality issues for accounting information systems' implementation: Systems, stakeholders, and organizational factors. Journal of Technology Research, 1, 111. 